\title{
KOSZUL SPACES
}

\author{
ALEXANDER BERGLUND
}

\begin{abstract}
We prove that a nilpotent space is both formal and coformal if and only if it is rationally homotopy equivalent to the derived spatial realization of a graded commutative Koszul algebra. We call such spaces Koszul spaces and show that the rational homotopy groups and the rational homology of iterated loop spaces of Koszul spaces can be computed by applying certain Koszul duality constructions to the cohomology algebra.
\end{abstract}

\section{INTRODUCTION}

In this paper we develop methods within the theory of Koszul duality for algebras over operads and apply these to prove results in rational homotopy theory. Our principal result, Theorem 1.2, is a characterization of spaces that are simultaneously rationally formal and coformal. We prove that such spaces are exactly the images of graded commutative Koszul algebras under Sullivan's derived spatial realization functor. Motivated by this we propose to call such spaces Koszul spaces. To demonstrate the usefulness of this characterization we prove that the rational homotopy groups, with Whitehead products, and the rational homology of any iterated loop space, with Pontryagin products and Browder brackets, of a Koszul space can be calculated as Koszul duals of the cohomology algebra over appropriate operads; see Theorem 1.3 and Theorem 1.4 .

A key technical result is Theorem 2.9, which should be of independent interest; it has as a by-product an intrinsic characterization of Koszul algebras (over a Koszul operad) in terms of formality of the bar construction. Such a characterization seems to have been generally accepted as true, but to our knowledge has not yet appeared in the literature.

Before we can give the precise statements of our main results, Theorems $1.2,1.3$ and 1.4. we need to recall some facts from rational homotopy theory and Koszul duality theory. Recall [6, 11,31,34 that the rational homotopy type of a connected nilpotent space $X$ of finite $\mathbb{Q}$-type is modeled algebraically by the Sullivan-de Rham commutative differential graded algebra $A_{P L}^{*}(X)$ with cohomology $\mathrm{H}^{*}(X ; \mathbb{Q})$, or alternatively by Quillen's differential graded Lie algebra $\lambda(X)$ with homology the rational homotopy groups $\pi_{*}(\Omega X) \otimes \mathbb{Q}$ with Samelson products 1

- $X$ is called formal if $A_{P L}^{*}(X)$ is quasi-isomorphic to $\mathrm{H}^{*}(X ; \mathbb{Q})$ as a commutative differential graded algebra.

Received by the editors November 18, 2011 and, in revised form, August 8, 2012.

2010 Mathematics Subject Classification. Primary 55P62; Secondary 16S37.

This work was supported by the Danish National Research Foundation (DNRF) through the Centre for Symmetry and Deformation.

${ }^{1}$ If $X$ is not simply connected, then Quillen's Lie algebra $\lambda(X)$ has to be modified somewhat; see 3 
- $X$ is called coformal if $\lambda(X)$ is quasi-isomorphic to $\pi_{*}(\Omega X) \otimes \mathbb{Q}$ as a differential graded Lie algebra.

We refer to 25] for a further discussion about formality and coformality. The contravariant functor $A_{P L}^{*}$ from spaces to commutative differential graded algebras induces an equivalence between the homotopy categories of connected nilpotent rational spaces of finite $\mathbb{Q}$-type and minimal algebras of finite type. The inverse is given by the spatial realization functor $\langle-\rangle$; see [6, 11, 34. By the derived spatial realization of a commutative differential graded algebra we mean the spatial realization of its minimal model. Finally, recall that a graded commutative algebra $A$ is called a Koszul algebra if it is generated by elements $x_{i}$ modulo certain quadratic relations

$$
\sum_{i, j} c_{i j} x_{i} x_{j}=0
$$

such that $\operatorname{Tor}_{s, t}^{A}(\mathbb{Q}, \mathbb{Q})=0$ for $s \neq t[30$. Here, the extra grading on Tor comes from the grading on $A$ given by wordlength in the generators $x_{i}$. The algebra $A$ is of finite type if there are finitely many generators $x_{i}$ in each homological degree. There is also a notion of a Koszul Lie algebra; see $₫ 2$.

Definition 1.1. A Koszul space is a space that is rationally homotopy equivalent to the derived spatial realization of a finite type graded commutative Koszul algebra.

Theorem 1.2. Let $X$ be a connected nilpotent space of finite $\mathbb{Q}$-type. The following are equivalent:

(1) $X$ is both formal and coformal.

(2) $X$ is formal and the cohomology $\mathrm{H}^{*}(X ; \mathbb{Q})$ is a Koszul algebra.

(3) $X$ is coformal and the rational homotopy $\pi_{*}(\Omega X) \otimes \mathbb{Q}$ is a Koszul Lie algebra.

(4) $X$ is a Koszul space.

The proof is given at the end of $\$ 3$ and depends on results from $\$ 2$ and 93 , Examples of Koszul spaces are discussed in \$5.

If $A$ is a graded commutative Koszul algebra, its Koszul dual Lie algebra $A^{!_{\mathscr{L}} \text { e }}$ is the free graded Lie algebra on generators $\alpha_{i}$, of homological degree $\left|\alpha_{i}\right|=\left|x_{i}\right|-1$, modulo the orthogonal relations. This means that a relation

$$
\sum_{i, j} \lambda_{i j}\left[\alpha_{i}, \alpha_{j}\right]=0
$$

holds if and only if

$$
\sum_{i, j}(-1)^{\left|x_{i}\right|\left|\alpha_{j}\right|} c_{i j} \lambda_{i j}=0
$$

whenever the coefficients $c_{i j}$ represent a relation among the generators $x_{i}$ for $A$. We prove the following theorem along with Theorem 1.2 at the end of $₫ 3$

Theorem 1.3. If $X$ is a Koszul space, then homotopy and cohomology are Koszul dual in the sense that there is an isomorphism of graded Lie algebras

$$
\pi_{*}(\Omega X) \otimes \mathbb{Q} \cong \mathrm{H}^{*}(X ; \mathbb{Q})^{!_{\mathscr{L} i e}} .
$$

Koszul duality between commutative algebras and Lie algebras is an instance of Koszul duality for algebras over Koszul operads, as introduced by Ginzburg and Kapranov [16]. Indeed, the commutative operad COom is a Koszul operad 
and its Koszul dual operad is the Lie operad $\mathscr{L} i e=\mathscr{C o m}$. In general, if $A$ is a Koszul algebra over a Koszul operad $\mathscr{A}$, then it has a Koszul dual $A^{!}$, which is an algebra over the Koszul dual operad $\mathscr{A}^{!}$. An important operad in this context is the little $n$-cubes operad $\mathscr{C}_{n}$ from the theory of iterated loop spaces [5, 20]. By a theorem of $\mathrm{F}$. Cohen, the homology $\mathrm{H}_{*}\left(\mathscr{C}_{n}\right)$ is isomorphic to the operad $e_{n}$ that governs 'Gerstenhaber $n$-algebras' [8]; see $\$ 4$. Getzler and Jones [15] proved that the operad $e_{n}$ is Koszul and that it is Koszul self-dual up to a suspension; $e_{n}^{!}=\Sigma^{n-1} e_{n}$. In particular, a Koszul $e_{n}$-algebra $A$ has a Koszul dual $e_{n}$-algebra $A^{!_{e_{n}}}$. The little $n$-cubes operad acts on $n$-fold loop spaces $\Omega^{n} X$, so $e_{n}$ acts on the homology $\mathrm{H}_{*}\left(\Omega^{n} X ; \mathbb{Q}\right)$. On the other hand, any commutative algebra, such as $\mathrm{H}^{*}(X ; \mathbb{Q})$, can be viewed as an $e_{n}$-algebra in a natural way; see $₫ 4$. In $\$ 4$ we prove the following result.

Theorem 1.4. If $X$ is an n-connected Koszul space, then loop space homology and cohomology are Koszul dual in the sense that there is an isomorphism of $e_{n}$-algebras

$$
\mathrm{H}_{*}\left(\Omega^{n} X ; \mathbb{Q}\right) \cong \mathrm{H}^{*}(X ; \mathbb{Q})^{!_{e_{n}}} .
$$

For formal spaces it is in principle possible to extract any rational homotopy invariant from the cohomology algebra. However, doing this in practice entails the non-trivial algebraic problem of constructing a minimal model for the cohomology. Our results imply that for spaces that are both formal and coformal, Koszul duality theory provides an explicit and direct way of extracting the rational homotopy groups and loop space homology from the cohomology.

Before getting into the proofs of Theorems 1.2, 1.3 and 1.4 we list some simple but interesting consequences. First, Theorem 1.2 settles in the positive a question of Papadima and Suciu [28, Question 8.2].

Corollary 1.5. Let $\mathcal{A}$ be an arrangement of hyperplanes in $\mathbb{C}^{\ell}$ and let $X=M(\mathcal{A})$ denote its complement. Let $\mathcal{A}^{k+1}$ denote the corresponding redundant subspace arrangement in $\mathbb{C}^{(k+1) \ell}$ and let $Y=M\left(\mathcal{A}^{k+1}\right)$. Then $Y$ is coformal if and only if $\mathrm{H}^{*}(X ; \mathbb{Q})$ is a Koszul algebra.

Proof. Both $X$ and $Y$ are formal (see [28, §1.5]), so if $Y$ is also coformal, then it follows from Theorem 1.2 that $\mathrm{H}^{*}(Y ; \mathbb{Q})$ is a Koszul algebra. From the rescaling formula

$$
\mathrm{H}^{*}(Y ; \mathbb{Q})=\mathrm{H}^{*}(X ; \mathbb{Q})[k]
$$

(see $[28, \S 1.3])$ it easily follows that $\mathrm{H}^{*}(X ; \mathbb{Q})$ is Koszul as well.

In 1965, Serre [33, IV-52] asked whether the Poincaré series

$$
\sum_{i \geq 0} \operatorname{dim}_{\mathbb{Q}} \mathrm{H}_{i}(\Omega X ; \mathbb{Q}) z^{i}
$$

of a simply connected finite CW-complex $X$ is always a rational function. This question remained open until a counterexample was constructed by Anick in 1982 [1,2]. It is interesting to note that such counterexamples cannot be Koszul spaces because of a certain relation between the Poincaré series of a Koszul algebra and its dual.

Corollary 1.6. If $X$ is a simply connected Koszul space with finitely generated rational cohomology algebra, then $\mathrm{H}_{*}(\Omega X ; \mathbb{Q})$ has rational Poincaré series. 
Proof. For a bigraded algebra $A$, consider the bigraded Poincaré series

$$
A(t, z)=\sum_{i, j} \operatorname{dim}_{\mathbb{Q}} A_{i}(j) z^{i} t^{j},
$$

where $t$ keeps track of the weight grading and $z$ keeps track of the homological grading. The bigraded Poincaré series of a Koszul algebra $A$ and its Koszul dual associative algebra $A^{!}$are related by the following formula:

$$
A^{!}(t, z)=A\left(-t z^{-1}, z\right)^{-1} \text {. }
$$

This can be seen, e.g., by refining the argument given in [18, Theorem 3.5.1]. In particular, $A^{!}(1, z)$ is rational if $A(t, z)$ is rational. It is well known that any finitely generated graded commutative algebra has rational Poincaré series (see e.g., [3, Theorem 11.1] for the strictly commutative case), so the claim follows from Theorem 1.4 with $n=1$.

Corollary 1.7. If $X$ is a Koszul space with finitely generated cohomology algebra, then the rational homotopy Lie algebra $\pi_{*}(\Omega X) \otimes \mathbb{Q}$ is finitely generated.

Proof. The Koszul dual Lie algebra of a finitely generated commutative Koszul algebra is finitely generated, so the claim follows from Theorem 1.3

Our results also shed new light on existing results. The following corollary recovers a well-known result due to F. Cohen [8], but our short proof using Koszul duality is new.

Corollary 1.8 (F. Cohen [8]). For any connected space $Y$, there is an isomorphism of $e_{n}$-algebras

$$
\mathrm{H}_{*}\left(\Omega^{n} \Sigma^{n} Y ; \mathbb{Q}\right) \cong e_{n}\left(\widetilde{\mathrm{H}}_{*}(Y ; \mathbb{Q})\right),
$$

where the right-hand side denotes the free $e_{n}$-algebra generated by the reduced homology of $Y$.

Proof. The $n$-fold suspension $\Sigma^{n} Y$ is an $n$-connected Koszul space whose cohomology $\mathrm{H}^{*}\left(\Sigma^{n} Y ; \mathbb{Q}\right)$ is a trivial algebra 'generated' by $\widetilde{\mathrm{H}}^{*}\left(\Sigma^{n} Y ; \mathbb{Q}\right) \cong s^{-n} \widetilde{\mathrm{H}}^{*}(Y ; \mathbb{Q})$. The Koszul dual $e_{n}$-algebra of a trivial $e_{n}$-algebra generated by a graded vector space $V$ is the free $e_{n}$-algebra $e_{n}\left(\left(s^{n} V\right)^{\vee}\right)$.

In view of Cohen's calculation of $\mathrm{H}_{*}\left(\Omega^{n} \sum^{n} Y ; \mathbb{F}_{p}\right)$, , it would be interesting to see to what extent Theorem 1.4 extends to finite coefficients. Here one would also need to take into consideration Araki-Kudo-Dyer-Lashof and Steenrod operations.

Relations between the Koszul property, formality and coformality have been studied in 28] and 29], but with a more restrictive definition of Koszul algebras; in 28,29, Koszul algebras are required to be generated in cohomological degree 1 . In [28. Example 4.10], an example is given of a space which is formal and coformal but whose cohomology algebra is not Koszul according to the more restrictive definition, namely $S^{1} \vee S^{2}$. The cohomology algebra is not Koszul according to the more restrictive definition, simply because it is not generated in cohomological degree 1 , but it is Koszul according to our definition, so this does not contradict Theorem 1.2. Theorem 1.2 and Theorem 1.3 together imply the main result of [29] in the nilpotent case.

Corollary 1.9 (Papadima-Yuzvinsky [29]). If $X$ is a formal nilpotent space, then $X$ is a rational $K(\pi, 1)$-space if and only if $\mathrm{H}^{*}(X ; \mathbb{Q})$ is Koszul and generated in cohomological degree 1 . 
Proof. If $X$ is formal and $\mathrm{H}^{*}(X ; \mathbb{Q})$ is Koszul, then $X$ is a Koszul space, and so by Theorem 1.3 we have that $\pi_{*}(\Omega X) \otimes \mathbb{Q} \cong \mathrm{H}^{*}(X ; \mathbb{Q})^{!} \mathscr{L L i e}$. If $\mathrm{H}^{*}(X ; \mathbb{Q})$ is generated in cohomological degree 1 , then $\mathrm{H}^{*}(X ; \mathbb{Q})^{!} \mathscr{L}_{i e}$ is generated in homological degree 0 , which forces it to be concentrated in degree 0 . Hence $\pi_{n}(X) \otimes \mathbb{Q}=0$ for $n>1$.

Conversely, for any differential graded Lie algebra $L$ that is concentrated in nonnegative homological degrees, there is a morphism $L \rightarrow \mathrm{H}_{0}(L)$, which is a quasiisomorphism if and only if $\mathrm{H}_{n}(L)=0$ for $n>0$. In particular, $L$ is coformal if it has homology concentrated in degree 0 . It follows that every rational $K(\pi, 1)$-space is coformal. Thus, if $X$ is simultaneously formal and a rational $K(\pi, 1)$, it is both formal and coformal, and hence the cohomology is Koszul by Theorem 1.2 .

It should be possible to extend our main results to the not necessarily nilpotent case so as to include [29, Theorem 5.1] as a special case. The main obstacle is the lack of a Lie model $\lambda(X)$, as well as an appropriate notion of coformality, for non-nilpotent spaces $X$.

We will end this introduction with a question. By Theorems 1.2 and 1.3 , if $X$ is a Koszul space, then $\mathrm{H}^{*}(X ; \mathbb{Q})$ and $\pi_{*}(\Omega X) \otimes \mathbb{Q}$ are both Koszul and they are Koszul dual to one another. What about the converse statement? Suppose that $X$ is a nilpotent space such that $\mathrm{H}^{*}(X ; \mathbb{Q})$ and $\pi_{*}(\Omega X) \otimes \mathbb{Q}$ are both Koszul and Koszul dual to one another. Then is $X$ necessarily a Koszul space? At the moment, we do not know of a counterexample. If true, this would give a characterization of Koszul spaces that did not contain any reference to the notions of formality or coformality.

\section{Koszul DUAlity For Algebras OVER Operads}

Koszul algebras were introduced by Priddy 30. Koszul duality for operads was developed by Ginzburg and Kapranov [16], and by Getzler and Jones [15. A modern comprehensive introduction to Koszul duality theory for operads can be found in the recent book [18 by Loday and Vallette. See also Fresse [13. Koszul duality for algebras over Koszul operads was introduced in [16] and has recently been developed further by Millès [21. In what follows, we will develop the theory needed for proving the main results of this section - Theorem 2.8, Theorem 2.9 and Corollary 2.10. We will freely use terminology from [18] without further reference.

2.1. Twisting morphisms, bar and cobar constructions. Let $\mathscr{C}$ be a coaugmented cooperad and $\mathscr{A}$ an augmented operad in chain complexes and let $\tau: \mathscr{C} \rightarrow$ $\mathscr{A}$ be an operadic twisting morphism. Let $C$ be a $\mathscr{C}$-coalgebra and let $A$ be an $\mathscr{A}$-algebra. A twisting morphism relative to $\tau$ is a map $\kappa: C \rightarrow A$ of degree 0 such that

$$
\partial(\kappa)+\tau * \kappa=0,
$$

where $\tau * \kappa$ is the composite map

$$
C \stackrel{\Delta}{\longrightarrow} \mathscr{C}(C) \stackrel{\tau(\kappa)}{\longrightarrow} \mathscr{A}(A) \stackrel{\mu}{\longrightarrow} A .
$$

Let $\mathrm{Tw}_{\tau}(C ; A)$ denote the set of twisting morphisms relative to $\tau$. It is a bifunctor $\mathscr{C}_{\text {coalg }}^{\text {op }} \times \mathscr{A}_{\text {alg }} \rightarrow$ Set. For a fixed $\mathscr{A}$-algebra $A$, the functor $\mathrm{Tw}_{\tau}(-; A)$ is represented by the bar construction $B_{\tau} A$, and for a fixed $\mathscr{C}$-coalgebra $C$, the functor $\operatorname{Tw}_{\tau}(C ;-)$ 
is represented by the cobar construction $\Omega_{\tau} C$; there are natural bijections

$$
\operatorname{Hom}_{\mathscr{A}}\left(\Omega_{\tau} C, A\right) \cong \operatorname{Tw}_{\tau}(C ; A) \cong \operatorname{Hom}_{\mathscr{C}}\left(C, B_{\tau} A\right) .
$$

In particular, the bar and cobar constructions form an adjoint pair of functors:

$$
\mathscr{C}_{\text {coalg }} \underset{B_{\tau}}{B_{\tau}} \mathscr{A}_{\text {alg }} .
$$

The bar construction is defined as the $\mathscr{C}$-coalgebra $B_{\tau} A=(\mathscr{C}(A), d+b)$, where $d$ is the internal differential of the chain complex $\mathscr{C}(A)$ and $b$ is the unique $\mathscr{C}$ coderivation making the diagram

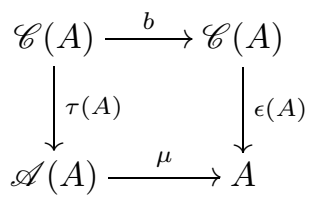

commute. The fact that $(d+b)^{2}=0$ is a consequence of the Maurer-Cartan equation for $\tau$.

The cobar construction is defined as the $\mathscr{A}$-algebra $\Omega_{\tau} C=(\mathscr{A}(C), d+\delta)$, where $d$ is the internal differential of $\mathscr{A}(C)$ and $\delta$ is the unique $\mathscr{A}$-derivation making the diagram

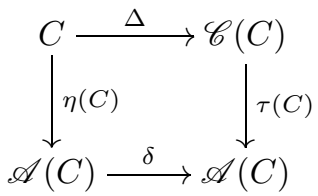

commute. The fact that $(d+\delta)^{2}=0$ is a consequence of the Maurer-Cartan equation for $\tau$.

The bijection (2.1) can be made explicit as follows. The unit and counit maps $\eta(C): C \rightarrow \mathscr{A}(C)$ and $\epsilon(A): \mathscr{C}(A) \rightarrow A$ define twisting morphisms $\iota: C \rightarrow \Omega_{\tau} C$ and $\pi: B_{\tau} A \rightarrow A$ called the universal twisting morphisms. Given a morphism of $\mathscr{C}$ coalgebras $\phi: C \rightarrow B_{\tau} A$ we get a twisting morphism $\kappa=\pi_{*}(\phi): C \rightarrow A$. Similarly, given a morphism of $\mathscr{A}$-algebras $\psi: \Omega_{\tau} C \rightarrow A$, we get a twisting morphism $\kappa=$ $\iota^{*}(\psi): C \rightarrow A$. Thus

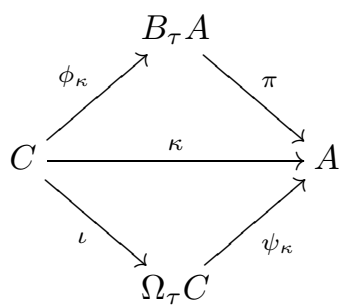

Conversely, given a twisting morphism $\kappa: C \rightarrow A$, there is an associated morphism of $\mathscr{C}$-algebras $\phi_{\kappa}: C \rightarrow B_{\tau} A$ given by the composite map

$$
C \stackrel{\Delta}{\longrightarrow} \mathscr{C}(C) \stackrel{\mathscr{C}(\kappa)}{\longrightarrow} \mathscr{C}(A)
$$


and there is an associated morphism of $\mathscr{A}$-algebras $\psi_{\kappa}: \Omega_{\tau} C \rightarrow A$ given by the composite map

$$
\mathscr{A}(C) \stackrel{\mathscr{A}(\kappa)}{\longrightarrow} \mathscr{A}(A) \stackrel{\mu}{\longrightarrow} A .
$$

The unit and counit of the adjunction give natural morphisms of $\mathscr{A}$-algebras and $\mathscr{C}$-coalgebras

$$
\Omega_{\tau} B_{\tau} A \rightarrow A, \quad C \rightarrow B_{\tau} \Omega_{\tau} C .
$$

The operadic twisting morphism $\tau: \mathscr{C} \rightarrow \mathscr{A}$ is an operadic Koszul twisting morphism if these are quasi-isomorphisms for all $C$ and $A$.

Definition 2.1. A twisting morphism $\kappa: C \rightarrow A$ relative to $\tau$ is a Koszul twisting morphism if the associated morphisms $\phi_{\kappa}: C \rightarrow B_{\tau} A$ and $\psi_{\kappa}: \Omega_{\tau} C \rightarrow A$ are quasiisomorphisms. We will denote the set of Koszul twisting morphisms relative to $\tau$ from $C$ to $A$ by $\operatorname{Kos}_{\tau}(C ; A)$.

In particular, the operadic twisting morphism $\tau: \mathscr{C} \rightarrow \mathscr{A}$ is Koszul if and only if the universal twisting morphisms $\pi: B_{\tau} A \rightarrow A$ and $\iota: C \rightarrow \Omega_{\tau} C$ are Koszul for all $C$ and $A$.

Definition 2.2. $\quad$ - A morphism of $\mathscr{A}$-algebras $\psi: A \rightarrow A^{\prime}$ is called a weak equivalence if it is a quasi-isomorphism.

- A morphism of $\mathscr{C}$-coalgebras $\phi: C \rightarrow C^{\prime}$ is called a weak equivalence if the associated morphism of $\mathscr{A}$-algebras $\Omega_{\tau} \phi: \Omega_{\tau} C \rightarrow \Omega_{\tau} C^{\prime}$ is a quasiisomorphism.

Every weak equivalence of $\mathscr{C}$-coalgebras is a quasi-isomorphism, but not conversely. A counterexample is given by the map of associative dg-coalgebras $B k[x] \rightarrow$ $B k\left[x, x^{-1}\right]$ induced by the algebra inclusion $k[x] \subseteq k\left[x, x^{-1}\right]$. The bar construction $B_{\tau}$ preserves but does not reflect quasi-isomorphisms. The cobar construction $\Omega_{\tau}$ reflects but does not preserve quasi-isomorphisms. However, the definition of weak equivalence is rigged so that the bar and cobar constructions both preserve and reflect weak equivalences. There are model category structures on $\mathscr{A}$-algebras and $\mathscr{C}$-coalgebras with these weak equivalences; see 35 .

Definition 2.3. (1) We say that two $\mathscr{A}$-algebras $A$ and $A^{\prime}$ are weakly equivalent and write $A \sim A^{\prime}$ if there is a zig-zag of weak equivalences of $\mathscr{A}$ algebras

$$
A \stackrel{\sim}{\longleftarrow} A_{1} \stackrel{\sim}{\longrightarrow} A_{2} \stackrel{\sim}{\sim} \ldots \stackrel{\sim}{\longrightarrow} A_{n} \stackrel{\sim}{\sim} A^{\prime} .
$$

We say that two $\mathscr{C}$-coalgebras $C$ and $C^{\prime}$ are weakly equivalent and write $C \sim C^{\prime}$ if they can be connected through a zig-zag of weak equivalences of $\mathscr{C}$-coalgebras.

(2) An $\mathscr{A}$-algebra $A$ is called formal if it is weakly equivalent to its homology, $A \sim \mathrm{H}_{*}(A)$. A $\mathscr{C}$-coalgebra $C$ is called formal if it is weakly equivalent to its homology $C \sim H_{*}(C)$.

Proposition 2.4. Let $\kappa: C \rightarrow A$ be a twisting morphism relative to an operadic Koszul twisting morphism $\tau: \mathscr{C} \rightarrow \mathscr{A}$. The following are equivalent:

(1) $\kappa$ is a Koszul twisting morphism.

(2) $\psi_{\kappa}: \Omega_{\tau} C \rightarrow A$ is a weak equivalence.

(3) $\phi_{\kappa}: C \rightarrow B_{\tau} A$ is a weak equivalence. 
Proof. Since $\tau$ is Koszul, the natural morphism of $\mathscr{A}$-algebras $\epsilon_{\tau}: \Omega_{\tau} B_{\tau} A \rightarrow A$ is a quasi-isomorphism. The composite morphism

$$
\Omega_{\tau} C \stackrel{\Omega_{\tau} \phi_{\kappa}}{\longrightarrow} \Omega_{\tau} B_{\tau} A \stackrel{\epsilon_{\tau}}{\longrightarrow} A
$$

is equal to $\psi_{\kappa}$. Therefore, $\psi_{\kappa}$ is a quasi-isomorphism if and only if $\Omega_{\tau} \phi_{\kappa}$ is.

An $\infty$-morphism $A \rightsquigarrow A^{\prime}$ between $\mathscr{A}$-algebras is a morphism of $\mathscr{C}$-coalgebras $B_{\tau} A \rightarrow B_{\tau} A^{\prime}$, and an $\infty$-morphism $C \rightsquigarrow C^{\prime}$ between $\mathscr{C}$-coalgebras is a morphism of $\mathscr{A}$-algebras $\Omega_{\tau} C \rightarrow \Omega_{\tau} C^{\prime}$. According to [18, Theorem 11.4.14], if $\mathscr{A}$ is a Koszul operad, then $A \sim A^{\prime}$ if and only if there exists an $\infty$-quasi-isomorphism $A \rightsquigarrow A^{\prime}$. From the bijections of (2.1) it is clear that $\operatorname{Tw}_{\tau}(C, A)$ is functorial with respect to $\infty$-morphisms. Furthermore, $\operatorname{Kos}_{\tau}(C, A)$ is functorial with respect to $\infty$-quasiisomorphisms. Since any $\infty$-quasi-isomorphism admits an inverse [18, Theorem 10.4.7], we get in particular the following result.

Proposition 2.5. Let $\kappa: C \rightarrow A$ be a Koszul twisting morphism relative to an operadic Koszul twisting morphism $\tau: \mathscr{C} \rightarrow \mathscr{A}$ where $\mathscr{A}$ is a Koszul operad. If $C \sim C^{\prime}$ and $A \sim A^{\prime}$, then there is a Koszul twisting morphism $\kappa^{\prime}: C^{\prime} \rightarrow A^{\prime}$.

2.2. Weight gradings and Koszul algebras over Koszul operads. A weight grading on an $\mathscr{A}$-algebra $A$ is a decomposition

$$
A=A(1) \oplus A(2) \oplus \cdots
$$

which is compatible with the $\mathscr{A}$-algebra structure in the sense that the structure map $\mu: \mathscr{A}(A) \rightarrow A$ is weight preserving. The bar construction $B_{\tau} A=(\mathscr{C}(A), d+b)$ is then bigraded by weight $\ell_{w}$ and bar length $\ell_{b}$. Since $A$ is concentrated in positive weight, the bar construction is concentrated in the region $\left\{\ell_{w} \geq \ell_{b}\right\}$. Let $\mathscr{D}_{b}=$ $\left\{\ell_{w}=\ell_{b}\right\}$ denote the diagonal. The coderivation $b$ preserves weight and decreases the bar length filtration. The subspace

$$
A^{\mathrm{i}}:=\mathscr{D}_{b} \cap \operatorname{ker}(b) \subseteq B_{\tau} A
$$

is naturally a $\mathscr{C}$-coalgebra and the inclusion map $A^{\mathrm{i}} \rightarrow B_{\tau} A$ is a morphism of $\mathscr{C}$-coalgebras.

Definition 2.6. We say that the weight grading on $A$ is a Koszul weight grading if the inclusion

$$
A^{\mathrm{i}} \rightarrow B_{\tau} A
$$

is a quasi-isomorphism. A Koszul $\mathscr{A}$-algebra is an $\mathscr{A}$-algebra $A$ that admits a Koszul weight grading. If $A$ is Koszul, then we call $A^{\text {i }}$ the Koszul dual $\mathscr{C}$-coalgebra.

Let $C$ be a $\mathscr{C}$-coalgebra. A weight grading on $C$ is a decomposition

$$
C=C(1) \oplus C(2) \oplus \cdots
$$

which is compatible with the coalgebra structure in the sense that $\Delta: C \rightarrow \mathscr{C}(C)$ preserves the weight. The cobar construction $\Omega_{\tau} C=(\mathscr{A}(C), d+\delta)$ is then bigraded by weight $\ell_{w}$ and cobar length $\ell_{c}$. Since $C$ is concentrated in positive weight, the cobar construction is concentrated in the region $\left\{\ell_{w} \geq \ell_{c}\right\}$ and we let $\mathscr{D}_{c}=\left\{\ell_{w}=\right.$ $\left.\ell_{c}\right\}$ denote the diagonal. The derivation $\delta$ preserves weight and increases the cobar length filtration. We define

$$
C^{\mathrm{i}}:=\mathscr{D}_{c} / \mathscr{D}_{c} \cap \operatorname{im}(\delta) .
$$


Then $C^{\mathrm{i}}$ is an $\mathscr{A}$-algebra and the projection

$$
f: \Omega_{\tau} C \rightarrow C^{\mathrm{i}}
$$

is a morphism of $\mathscr{A}$-algebras.

Definition 2.7. We say that a weight grading on $C$ is a Koszul weight grading if the projection map $f: \Omega_{\tau} C \rightarrow C^{i}$ is a quasi-isomorphism. A Koszul $\mathscr{C}$-coalgebra is a $\mathscr{C}$-coalgebra $C$ that admits a Koszul weight grading. If $C$ is Koszul, then we call $C^{\mathrm{i}}$ the Koszul dual $\mathscr{A}$-algebra.

We will call an operadic twisting morphism $\tau: \mathscr{C} \rightarrow \mathscr{A}$ binary if it vanishes outside arity 2 .

Theorem 2.8. Let $\kappa: C \rightarrow A$ be a Koszul twisting morphism relative to a binary Koszul twisting morphism $\tau: \mathscr{C} \rightarrow \mathscr{A}$. If $C$ and $A$ have trivial differentials, then there are Koszul weight gradings on $C$ and $A$ such that $\phi_{\kappa}: C \rightarrow B_{\tau} A$ maps $C$ isomorphically onto $A^{\mathrm{i}}$ and $\psi_{\kappa}: \Omega_{\tau} C \rightarrow A$ factors through an isomorphism $C^{\mathrm{i}} \rightarrow A$.

Proof. Since $A$ has trivial differential, the quasi-isomorphism $\psi_{\kappa}: \Omega_{\tau} C \rightarrow A$ is surjective and induces an isomorphism $\mathrm{H}_{*}\left(\Omega_{\tau} C\right) \cong A$. Since $C$ has trivial differential and since $\tau$ is binary, the derivation $\delta$ on $\Omega_{\tau} C$ increases the cobar length by exactly 1. Therefore we can introduce a weight grading on the homology $H=\mathrm{H}_{*}\left(\Omega_{\tau} C\right)$ by

$$
H(p)=\frac{\operatorname{ker}\left(\delta: \mathscr{A}(p) \otimes_{\Sigma_{p}} C^{\otimes p} \rightarrow \mathscr{A}(p+1) \otimes_{\Sigma_{p+1}} C^{\otimes p+1}\right)}{\operatorname{im}\left(\delta: \mathscr{A}(p-1) \otimes_{\Sigma_{p-1}} C^{\otimes p-1} \rightarrow \mathscr{A}(p) \otimes_{\Sigma_{p}} C^{\otimes p}\right)},
$$

which we transport to a weight grading on $A$ via the isomorphism $\mathrm{H}_{*}\left(\Omega_{\tau} C\right) \cong A$. It follows that $A(p)$ is the image of $\mathscr{A}(p) \otimes_{\Sigma_{p}} C^{\otimes p}$ under $\psi_{\kappa}$.

Similarly, since $C$ has trivial differential, the quasi-isomorphism $\phi_{\kappa}: C \rightarrow B_{\tau} A$ is injective and induces an isomorphism $C \cong \mathrm{H}_{*}\left(B_{\tau} A\right)$. Since $A$ has trivial differential and since $\tau$ is binary, the coderivation $b$ on the bar construction decreases the bar length by exactly 1 and as before we can introduce a weight grading on the homology $\mathrm{H}_{*}\left(B_{\tau} A\right)$, which we transport to $C$. It follows that the induced weight grading on $C$ is given by $C(p)=\phi_{\kappa}^{-1}\left(\mathscr{C}(p) \otimes_{\Sigma_{p}} A^{\otimes p}\right)$.

We will now verify that the weight gradings thus constructed are Koszul by proving that the image of $\phi_{\kappa}$ is equal to $A^{\mathrm{i}}=\mathscr{D}_{b} \cap \operatorname{ker}(b)$ and that $\psi_{\kappa}$ factors through an isomorphism $C^{\mathrm{i}}=\mathscr{D}_{c} / \mathscr{D}_{c} \cap \operatorname{im}(\delta) \cong A$. To this end, first note that $\kappa: C \rightarrow A$ vanishes on elements of weight different from 1 and has image concentrated in weight 1. This follows from the factorization of $\kappa$ as in the diagram (2.2). Indeed, the weight grading on $C$ is inherited from the bar length grading on $B_{\tau} A$ and $\pi: B_{\tau} A \rightarrow A$ vanishes outside bar length 1. Similarly, the image of $\iota: C \rightarrow \Omega_{\tau} C$ is contained in the cobar length 1 component, whose image under $\psi_{\kappa}: \Omega_{\tau} C \rightarrow A$ is contained in $A(1)$. Recall that $\phi_{\kappa}$ is the composite

$$
C \stackrel{\Delta}{\longrightarrow} \mathscr{C}(C) \stackrel{\mathscr{C}(\kappa)}{\longrightarrow} \mathscr{C}(A) .
$$

Since the image of $\kappa$ is contained in weight 1 , this shows that $\operatorname{im}\left(\phi_{\kappa}\right) \subseteq \mathscr{D}_{b}$. Since $C$ has trivial differential, $\operatorname{im}\left(\phi_{\kappa}\right) \subseteq \operatorname{ker}(b)$. Thus, $\operatorname{im}\left(\phi_{\kappa}\right) \subseteq \mathscr{D}_{b} \cap \operatorname{ker}(b)$. That we have equality follows because $\phi_{\kappa}$ is a quasi-isomorphism. Indeed, if $x \in \mathscr{D}_{b} \cap \operatorname{ker}(b)$, then the homology class of $x$ must be the image of the homology class of some $c \in C$, that is to say, $\phi_{\kappa}(c)-x \in \operatorname{im}(b)$. But we also have that $\phi_{\kappa}(c)-x \in \mathscr{D}_{b}$. Since $\mathscr{D}_{b} \cap \operatorname{im}(b)=0$ (which follows from the fact that $\ell_{w} \geq \ell_{b}$ ) we get $\phi_{\kappa}(c)=x$. Thus, 
the quasi-isomorphism $\phi_{\kappa}: C \rightarrow B_{\tau} A$ maps isomorphically onto $A^{\mathrm{i}}$, so indeed the weight grading on $A$ is Koszul.

Recall that $\psi_{\kappa}$ is the composite

$$
\mathscr{A}(C) \stackrel{\mathscr{A}(\kappa)}{\longrightarrow} \mathscr{A}(A) \stackrel{\mu}{\longrightarrow} A .
$$

Since $\kappa$ vanishes outside weight 1 , this shows that $\psi_{\kappa}$ vanishes outside the diagonal $\mathscr{D}_{c}$. Moreover, since $A$ has trivial differential, $\psi_{\kappa}$ factors through $C^{\mathrm{i}}=\mathscr{D}_{c} / \mathscr{D}_{c} \cap$ $\operatorname{im}(\delta) \rightarrow A$. Since $\psi_{\kappa}$ is surjective this map is necessarily surjective. That it is injective follows because $\psi_{\kappa}$ is a quasi-isomorphism. Indeed, if $x \in \mathscr{D}_{c} \cap \operatorname{ker}\left(\psi_{\kappa}\right)$, then $\delta(x)=0$ since $\delta\left(\mathscr{D}_{c}\right)=0$ as $\ell_{w} \geq \ell_{c}$ and $\delta$ preserves weight but increases cobar length. Thus, the homology class of $x$ gets mapped to zero under $\psi_{\kappa}$, which implies that $x \in \operatorname{im}(\delta)$ as $\psi_{\kappa}$ is a quasi-isomorphism. Thus, the quasi-isomorphism

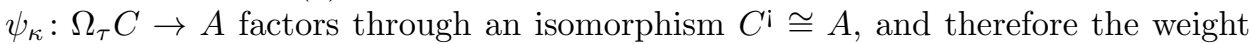
grading on $C$ is Koszul.

Theorem 2.9. Let $\kappa: C \rightarrow A$ be a Koszul twisting morphism relative to a binary Koszul twisting morphism $\tau: \mathscr{C} \rightarrow \mathscr{A}$ where $\mathscr{A}$ is a Koszul operad. The following are equivalent:

(1) $C$ and $A$ are formal.

(2) A is formal and $\mathrm{H}_{*}(A)$ is a Koszul $\mathscr{A}$-algebra.

(3) $C$ is formal and $\mathrm{H}_{*}(C)$ is a Koszul $\mathscr{C}$-coalgebra.

Proof. (1) $\Rightarrow(2)$,(3): If $C \sim \mathrm{H}_{*}(C)$ and $A \sim \mathrm{H}_{*}(A)$, then there is a Koszul twisting morphism $\kappa^{\prime}: \mathrm{H}_{*}(C) \rightarrow \mathrm{H}_{*}(A)$ by Proposition 2.5 . By Theorem 2.8 this implies that $\mathrm{H}_{*}(C)$ and $\mathrm{H}_{*}(A)$ are Koszul and Koszul dual to each other.

$(2) \Rightarrow(1)$ : We need to prove that $C$ is formal. Since $A$ is formal we may assume that $A=\mathrm{H}_{*}(A)$ by Proposition 2.5. Then we have a weak equivalence of $\mathscr{C}$ coalgebras $C \rightarrow B_{\tau} A$. Since $A=\mathrm{H}_{*}(A)$ is assumed to be Koszul there is a weak equivalence $\mathrm{H}_{*}\left(B_{\tau} A\right) \rightarrow B_{\tau} A$. So we get a zig-zag of weak equivalences $C \rightarrow$ $B_{\tau} A \leftarrow \mathrm{H}_{*}\left(B_{\tau} A\right)$, showing that $C$ is formal.

The proof of the implication (3) $\Rightarrow(1)$ is similar.

Corollary 2.10. Let $\mathscr{A}$ be a binary Koszul operad with Koszul dual cooperad $\mathscr{C}$ and let $\tau: \mathscr{C} \rightarrow \mathscr{A}$ be the associated operadic Koszul twisting morphism.

(1) An $\mathscr{A}$-algebra $A$ with zero differential is Koszul if and only if the bar construction $B_{\tau} A$ is formal as a $\mathscr{C}$-coalgebra.

(2) A $\mathscr{C}$-coalgebra $C$ with zero differential is Koszul if and only if the cobar construction $\Omega_{\tau} C$ is formal as an $\mathscr{A}$-algebra.

If $\mathscr{A}$ is a Koszul operad with Koszul dual cooperad $\mathscr{C}$, then the Koszul dual operad is defined by $\mathscr{A}^{!}=(\Sigma \mathscr{C})^{\vee}$. If $A$ is a Koszul $\mathscr{A}$-algebra with Koszul dual $\mathscr{C}$-coalgebra $C$, then the Koszul dual $\mathscr{A}^{!}$-algebra is defined to be the weight graded algebra $\mathscr{A}^{!}$-algebra $A^{!}$with $A^{!}(p)=(s C(p))^{\vee}$.

Theorem 2.11. Let $A$ be a finite type Koszul algebra over a finite type binary Koszul operad $\mathscr{A}$. Then $A$ is quadratic, that is, $A$ has a presentation of the form

$$
A=\mathscr{A}(V) /(R)
$$


where $R \subseteq \mathscr{A}(V)(2)=\mathscr{A}(2) \otimes_{\Sigma_{2}} V^{\otimes 2}$. Furthermore, the Koszul dual $\mathscr{A} !$-algebra $A^{!}$is also Koszul and has quadratic presentation

$$
A^{!}=\mathscr{A}^{!}\left((s V)^{\vee}\right) /\left(R^{\perp}\right)
$$

where $R^{\perp} \subseteq \mathscr{A}^{!}\left((s V)^{\vee}\right)(2)$ is the annihilator of $R \subseteq \mathscr{A}(V)(2)$ with respect to the induced pairing of degree 2 :

$$
\langle,\rangle: \mathscr{A}^{!}\left((s V)^{\vee}\right)(2) \otimes \mathscr{A}(V)(2) \rightarrow \mathbb{Q} .
$$

Proof. Theorem 2.8 identifies $A$ with $C^{\mathrm{i}}=\mathscr{D}_{c} / \mathscr{D}_{c} \cap \operatorname{im}(\delta)$ where $C=A^{\mathrm{i}}$. The diagonal $\mathscr{D}_{c} \subseteq \Omega_{\tau} C$ may be identified with the free $\mathscr{A}$-algebra $\mathscr{A}(V)$ where $V=$ $C(1)$ is the weight 1 component of $C$. We need to identify the generators of the $\mathscr{A}$ ideal $\mathscr{D}_{c} \cap \operatorname{im}(\delta) \subseteq \mathscr{D}_{c}=\mathscr{A}(V)$. By definition, the map $\delta$ is the unique $\mathscr{A}$-derivation that makes the diagram

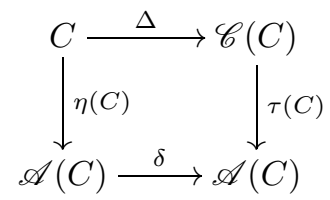

commute. This implies that the $\mathscr{A}$-ideal $\mathscr{D}_{c} \cap \operatorname{im}(\delta)$ is generated by the image of the map

$$
C \stackrel{\Delta}{\longrightarrow} \mathscr{C}(C) \stackrel{\tau(C)}{\longrightarrow} \mathscr{A}(C)
$$

intersected with the diagonal. But if $\mathscr{A}$ is binary, the operadic twisting morphism $\tau$ vanishes outside arity 2 . Therefore, this image is contained in the subspace $\mathscr{A}(2) \otimes_{\Sigma_{2}} V^{\otimes 2}$. This proves the first part of the theorem. The fact that $A^{!}$is Koszul follows because its bar construction can be identified, up to a shift, with the weight graded dual of the cobar construction on $C$.

To prove the statement about the orthogonal presentation for $A^{!}$, first note that $\kappa: C \rightarrow A$ restricts to an isomorphism of weight 1 components; $\kappa(1): C(1) \cong A(1)$. We identify these components and write $V$ for both. By definition, the surjective morphism of $\mathscr{A}$-algebras $\psi_{\kappa}: \mathscr{A}(C) \rightarrow A$ is the composite

$$
\mathscr{A}(C) \stackrel{\mathscr{A}(\kappa)}{\longrightarrow} \mathscr{A}(A) \stackrel{\mu}{\longrightarrow} A
$$

It vanishes outside the diagonal $\mathscr{D}_{c}=\mathscr{A}(V)$ and we also use $\mu$ to denote the induced surjective morphism of $\mathscr{A}$-algebras $\mathscr{A}(V) \rightarrow A$. Similarly, by definition the injective morphism of $\mathscr{C}$-coalgebras $\phi_{\kappa}: C \rightarrow \mathscr{C}(A)$ is the composite

$$
C \stackrel{\Delta}{\longrightarrow} \mathscr{C}(C) \stackrel{\mathscr{C}(\kappa)}{\longrightarrow} \mathscr{C}(A) .
$$

It has image $\mathscr{D}_{b} \cap$ ker $b$ and in particular it factors through an injective morphism of $\mathscr{C}$-coalgebras $C \rightarrow \mathscr{D}_{b}=\mathscr{C}(V)$ that we will also denote by $\Delta$. By inspecting the definitions of the bar and cobar differentials $b$ and $\delta$ we have the following 
commutative diagram with exact rows in weight 2 :

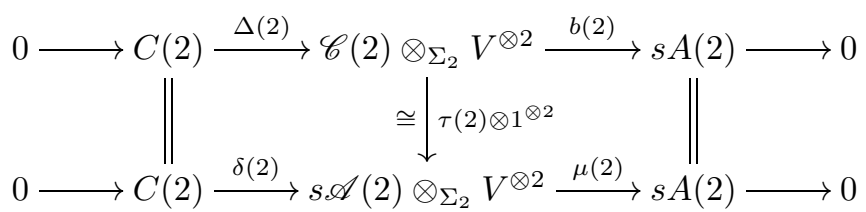

The injective morphism of weight graded $\mathscr{C}$-coalgebras $\Delta: C \rightarrow \mathscr{C}(V)$ dualizes (weight-wise) to a surjective morphism of $\mathscr{A}^{!}$-algebras

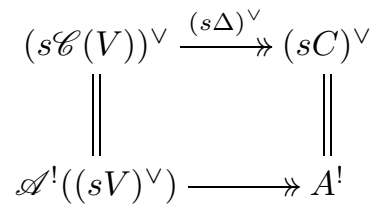

Since $A^{!}$is Koszul the kernel of this morphism is generated in weight 2 as an

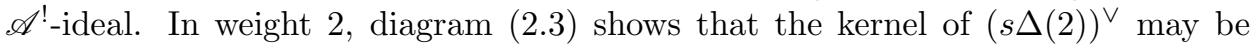
identified with $R^{\perp}$, since the kernel of $\mu(2)$ is $R$ by definition.

Let us be more explicit about the relation between $R$ and $R^{\perp}$. Let $W=(s V)^{\vee}$. The pairing $\langle\rangle:, W \otimes V \rightarrow \mathbb{Q}$ of degree 1 extends to a pairing of degree 2 ,

$$
\langle,\rangle:\left(\mathscr{A}^{!}(2) \otimes_{\Sigma_{2}} W^{\otimes 2}\right) \otimes\left(\mathscr{A}(2) \otimes_{\Sigma_{2}} V^{\otimes 2}\right) \rightarrow \mathbb{Q},
$$

defined by

$$
\langle(f ; \alpha, \beta),(\mu ; x, y)\rangle=(-1)^{\epsilon}\langle f, \mu\rangle\langle\alpha, x\rangle\langle\beta, y\rangle+(-1)^{\eta}\langle f \tau, \mu\rangle\langle\beta, x\rangle\langle\alpha, y\rangle,
$$

where the signs are given by

$$
\begin{gathered}
\epsilon=|\mu|(|\alpha|+|\beta|)+|\beta||x|+|\alpha|+|x|, \\
\eta=|\mu|(|\alpha|+|\beta|)+|\alpha||\beta|+|\alpha||x|+|\beta|+|x| .
\end{gathered}
$$

Every Koszul algebra over a binary Koszul operad is quadratic, but it is well known that not every quadratic algebra is Koszul. However, given a quadratic $\mathscr{A}$ algebra $A$, the orthogonal presentation defines a quadratic $\mathscr{A}^{!}$-algebra $A^{!}$and there is an associated twisting morphism $\kappa: A^{\mathrm{i}}=\left(s A^{!}\right)^{\vee} \rightarrow A$. There is a natural chain complex associated to $\kappa$ called the Koszul complex, which is acyclic if and only if $\kappa$ is a Koszul twisting morphism. This is a useful technique used to prove that a given quadratic algebra is Koszul. We refer to 21] for details.

\section{Rational homotopy theory and Koszul DUALity}

The rational homotopy type of a simply connected space $X$ of finite $\mathbb{Q}$-type is modeled in Sullivan's approach 34 by a commutative differential graded algebra $A_{P L}^{*}(X)$ with cohomology $\mathrm{H}^{*}(X ; \mathbb{Q})$ and in Quillen's approach 31 by a differential graded Lie algebra $\lambda(X)$ with homology $\pi_{*}(\Omega X) \otimes \mathbb{Q}$. See also [11.

There is an operadic Koszul twisting morphism $\tau: \mathscr{C o m}^{\vee} \rightarrow \Sigma \mathscr{L} i$ e where $\mathscr{C} o \mathrm{~m}^{\vee}$ is the cooperad whose coalgebras are non-counital cocommutative coalgebras and $\Sigma \mathscr{L}$ ie is the operad whose algebras are suspensions of Lie algebras. The associated bar and cobar constructions are related to the classical Quillen functors [11,23, 31]

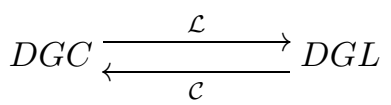


as follows. For a $\operatorname{dgc} C$ and a $\operatorname{dgl} L$,

$$
\Omega_{\tau}(\bar{C})=s \mathcal{L}(C), \quad B_{\tau}(s L)=\mathbb{Q} \oplus \mathcal{C}(L) .
$$

The Baues-Lemaire conjecture [4, Conjecture 3.5], proved by Majewski [19] (see also [11, Theorem 26.5]), can be expressed in the language of Koszul duality theory in the following way:

Quillen's and Sullivan's approaches to rational homotopy theory are Koszul dual to one another under the Koszul duality between the commutative and the Lie operad.

A more precise statement is the following.

Theorem 3.1 (Majewski [19). Let $X$ be a simply connected space of finite $\mathbb{Q}$-type and let $M_{X}$ be its minimal model. There is a quasi-isomorphism of differential graded Lie algebras

$$
\mathcal{L}\left(M_{X}^{\vee}\right) \stackrel{\sim}{\rightarrow} \lambda(X) .
$$

Whereas Sullivan's approach extends to nilpotent spaces, Quillen only defined his Lie model $\lambda(X)$ for simply connected spaces $X$. However, if $X$ is nilpotent and of finite $\mathbb{Q}$-type, then its minimal model $M_{X}$ is of finite type and can therefore be dualized to a differential graded coalgebra $C_{X}=M_{X}^{\vee}$. The Lie algebra $\mathcal{L}\left(C_{X}\right)$ may serve as a replacement for the Quillen model, as justified by Theorem 3.1. We refer the reader to Neisendorfer's work [23] for further details.

Theorem 3.2 (See [23, Proposition 8.2, 8.3]). Let $X$ be a nilpotent space of finite $\mathbb{Q}$-type and let $M_{X}$ be its minimal model. Then there is an isomorphism of graded Lie algebras

$$
\mathrm{H}_{\geq 1} \mathscr{L}\left(M_{X}^{\vee}\right) \cong \pi_{\geq 1}(\Omega X) \otimes \mathbb{Q} .
$$

Furthermore, there is an isomorphism of Lie algebras

$$
\mathrm{H}_{0} \mathscr{L}\left(M_{X}^{\vee}\right) \cong l\left(\pi_{1}(X) \otimes \mathbb{Q}\right),
$$

where the right-hand side is the Lie algebra of the Malcev completion of the fundamental group of $X$ 31.

Remark 3.3. As stated, Theorem 3.2 says nothing about the interpretation of the Lie bracket

$$
\mathrm{H}_{0}\left(\mathscr{L}\left(M_{X}^{\vee}\right)\right) \otimes \mathrm{H}_{n}\left(\mathscr{L}\left(M_{X}^{\vee}\right)\right) \rightarrow \mathrm{H}_{n}\left(\mathscr{L}\left(M_{X}^{\vee}\right)\right)
$$

for $n \geq 1$, but it is natural to expect that it corresponds to the action of $\pi_{1}(X)$ on the higher homotopy groups.

Proof of Theorem 1.2 and Theorem 1.3. Theorem 1.3 and the equivalence of (1), (2) and (3) follow directly by combining Theorem [2.9, Theorem 3.1 and Theorem 3.2. The equivalence (2) $\Leftrightarrow$ (4) follows from the Sullivan-de Rham Equivalence Theorem [6, Theorem 9.4] and the Localization Theorem [6, Theorem 11.2]: If $X$ is connected, nilpotent and of finite $\mathbb{Q}$-type, then the map $X \rightarrow\left\langle M_{X}\right\rangle$ is a rational homotopy equivalence, where $M_{X}$ is the minimal model for $X$. If $X$ is formal and $\mathrm{H}^{*}(X ; \mathbb{Q})$ is Koszul, then the minimal model for $X$ is also a minimal model for the cohomology, and hence $\left\langle M_{X}\right\rangle$ represents the derived spatial realization of the Koszul algebra $\mathrm{H}^{*}(X ; \mathbb{Q})$. Conversely, if $X$ is rationally homotopy equivalent to the derived spatial realization of a Koszul algebra $A$, this means that $X \sim_{\mathbb{Q}}\left\langle M_{A}\right\rangle$, where $M_{A}$ is the minimal model for the Koszul algebra $A$. By [6, Theorem 9.4], 
this implies that $M_{A}$ is also the minimal model for $X$, and since $M_{A} \stackrel{\sim}{\rightarrow} A$ this implies that $X$ is formal and that $\mathrm{H}^{*}(X ; \mathbb{Q}) \cong A$.

\section{Koszul Duality for Gerstenhaber $n$-Algebras and Rational HOMOLOGY OF $n$-FOLD LOOP SPACES}

A Gerstenhaber $n$-algebra is a graded vector space $A$ together with two binary operations

$$
\mu: A_{p} \otimes A_{q} \rightarrow A_{p+q}, \quad \lambda: A_{p} \otimes A_{q} \rightarrow A_{p+q+n-1},
$$

such that for all $x, y, z \in A$, writing $x y=\mu(x \otimes y)$ and $[x, y]=\lambda(x \otimes y)$,

$$
\begin{gathered}
x y=(-1)^{|x||y|} y x, \quad(x y) z=x(y z), \\
{[x, y]=-(-1)^{(|x|+n-1)(|y|+n-1)}[y, x],} \\
{[x,[y, z]]=[[x, y], z]+(-1)^{(|x|+n-1)(|y|+n-1)}[y,[x, z]],} \\
{[x, y z]=[x, y] z+(-1)^{(|x|+n-1)|y|} y[x, z] .}
\end{gathered}
$$

Theorem 4.1 (F. Cohen 8). The homology of the little $n$-cubes operad $\mathscr{C}_{n}$ is isomorphic to the operad $e_{n}$ of Gerstenhaber n-algebras. In particular, the homology of any $\mathscr{C}_{n}$-algebra has the structure of an $e_{n}$-algebra.

The prototypical example of a $\mathscr{C}_{n}$-algebra is the $n$-fold loop space $\Omega^{n} X$ of a based topological space $X\left[5,20\right.$. The homology $\mathrm{H}_{*}\left(\Omega^{n} X ; \mathbb{Q}\right)$ is an $e_{n}$-algebra where $\mu$ is the Pontryagin product and $\lambda$ the Browder bracket. Any graded commutative algebra $A$ may be viewed as an $e_{n}$-algebra by setting $\lambda=0$.

Theorem 4.2 (Getzler-Jones [15). The operad $e_{n}$ is Koszul and it is Koszul selfdual up to a suspension; $e_{n}^{!}=\Sigma^{n-1} e_{n}$.

If $A$ is a Koszul $e_{n}$-algebra, then the Koszul dual $A^{!}$as defined in 92 is an algebra over $e_{n}^{!}=\Sigma^{n-1} e_{n}$. In order to get an algebra over $e_{n}$ again, we define the Koszul dual $e_{n}$-algebra of $A$ to be the desuspension $A^{! e_{n}}=s^{1-n} A^{!}$.

Proposition 4.3. Let $A$ be a commutative algebra viewed as an $e_{n}$-algebra with trivial Lie bracket. Then $A$ is Koszul as a commutative algebra if and only if it is Koszul as an $e_{n}$-algebra. In this situation there is an isomorphism of $e_{n}$-algebras:

$$
A^{!_{e n}} \cong \Lambda\left(s^{1-n} A^{!_{\mathscr{L} i e}}\right) .
$$

Proof. This is a consequence of the decomposition of operads $e_{n}=\mathscr{C} o m \circ \mathscr{L i}_{n-1}$, where $\mathscr{L}_{i e_{n-1}}=\Sigma^{n-1} \mathscr{L} i e$; see [18, 13.3.14]. Since the Lie algebra structure on $A$ is trivial, the $e_{n}$ bar construction of $A$ is isomorphic to

$$
B_{e_{n}}(A) \cong \mathscr{L i}_{n-1}^{\mathrm{i}}\left(B_{\mathscr{C} o m}(A)\right) .
$$

It follows that $B_{e_{n}}(A)$ is formal as an $e_{n}^{i}$-coalgebra if and only if $B_{\mathscr{C} o m}(A)$ is formal as a $\mathscr{C} o m^{\mathrm{i}}$-coalgebra. This proves the first part of the proposition. For the second part, simply observe that $\mathscr{L} i e_{n-1}^{!}=\Sigma^{n-1} \mathscr{C} o m$.

Proposition 4.4. For any $n$-connected space $X$ there is an isomorphism of $e_{n^{-}}$ algebras,

$$
\mathrm{H}_{*}\left(\Omega^{n} X ; \mathbb{Q}\right) \cong \Lambda\left(s^{-n} \pi_{*}(X) \otimes \mathbb{Q}\right),
$$

where the Lie bracket on the right-hand side is induced by Whitehead products on the homotopy groups of $X$. 
Proof. By the Milnor-Moore theorem [22, p. 263], since $\Omega^{n} X$ is connected the Hurewicz homomorphism

$$
h: \pi_{*}\left(\Omega^{n} X\right) \otimes \mathbb{Q} \rightarrow \mathrm{H}_{*}\left(\Omega^{n} X ; \mathbb{Q}\right)
$$

induces an isomorphism of graded algebras between $\mathrm{H}_{*}\left(\Omega^{n} X ; \mathbb{Q}\right)$ and the universal enveloping algebra of the graded Lie algebra $\pi_{*}\left(\Omega^{n} X\right) \otimes \mathbb{Q}$ with the Samelson product. But for $n \geq 2$, the Samelson product on $\pi_{*}\left(\Omega^{n} X\right)$ is trivial, so the universal enveloping algebra is a free graded commutative algebra and we get that the Hurewicz homomorphism induces an isomorphism of graded algebras:

$$
\Lambda\left(\pi_{*}\left(\Omega^{n} X\right) \otimes \mathbb{Q}\right) \stackrel{\cong}{\rightrightarrows} \mathrm{H}_{*}\left(\Omega^{n} X ; \mathbb{Q}\right) .
$$

To finish the proof we note that under the identification $\pi_{*}\left(\Omega^{n} X\right)=s^{-n} \pi_{*}(X)$, the Hurewicz homomorphism takes Whitehead products in $\pi_{*}(X)$ to Browder brackets in $\mathrm{H}_{*}\left(\Omega^{n} X ; \mathbb{Q}\right)$ [8, p. 215 , p. 318$]$.

Proof of Theorem 1.4. The proof consists in assembling three facts:

- For any Koszul space $X$ there is an isomorphism of graded Lie algebras

$$
s^{-1} \pi_{*}(X) \otimes \mathbb{Q} \cong \mathrm{H}^{*}(X ; \mathbb{Q})^{!_{\mathscr{L} i e}}
$$

where the Lie bracket on the left-hand side is induced by Whitehead products on $\pi_{*}(X)$ (Theorem 1.3).

- For any commutative Koszul algebra $A$ viewed as an $e_{n}$-algebra with trivial Lie bracket there is an isomorphism of $e_{n}$-algebras

$$
A^{!_{e_{n}}} \cong \Lambda\left(s^{1-n} A^{!_{\mathscr{L i e}}}\right)
$$

(Proposition 4.3).

- For any $n$-connected space $X$ there is an isomorphism of $e_{n}$-algebras

$$
\mathrm{H}_{*}\left(\Omega^{n} X ; \mathbb{Q}\right) \cong \Lambda\left(s^{-n} \pi_{*}(X) \otimes \mathbb{Q}\right)
$$

where the Lie bracket on the right-hand side is induced by Whitehead products on $\pi_{*}(X)$ (Proposition 4.4).

\section{Examples of Koszul spaces}

Example 5.1. Spheres. The first example of a Koszul space is the sphere $S^{n}$, $n \geq 1$. Spheres are formal, and the cohomology algebra $\mathrm{H}^{*}\left(S^{n} ; \mathbb{Q}\right)$ is generated by a class $x$ of cohomological degree $n$ modulo the relation $x^{2}=0$. The Koszul dual Lie algebra is the free graded Lie algebra $\mathbb{L}(\alpha)$ on a generator $\alpha$ of homological degree $n-1$. Hence,

$$
\pi_{*}\left(\Omega S^{n}\right) \otimes \mathbb{Q} \cong \mathbb{L}(\alpha)=\left\{\begin{array}{cc}
\langle\alpha,[\alpha, \alpha]\rangle_{\mathbb{Q}}, & n \text { even } \\
\langle\alpha\rangle_{\mathbb{Q}}, & n \text { odd }
\end{array}\right.
$$

This gives a new perspective on Serre's 32 classical result that $\pi_{i}\left(S^{n}\right) \otimes \mathbb{Q}=\mathbb{Q}$ for $i=n$, and $i=2 n-1$ if $n$ is even, and $\pi_{i}\left(S^{n}\right) \otimes \mathbb{Q}=0$ otherwise.

Example 5.2. Suspensions. More generally, the suspension $\Sigma X$ of any connected space $X$ is a Koszul space. A suspension is formal (indeed rationally homotopy equivalent to a wedge of spheres; see [11, Theorem 24.5]) and the cohomology $\mathrm{H}^{*}(\Sigma X ; \mathbb{Q})$ is isomorphic to the trivial algebra $\Lambda(V) /\left(\Lambda^{2} V\right)$, where $V=\widetilde{\mathrm{H}}^{*}(\Sigma X ; \mathbb{Q})$ 
is the reduced cohomology of $\Sigma X$. The Koszul dual is the free graded Lie algebra on the reduced homology of $X$,

$$
\pi_{*}(\Omega \Sigma X) \otimes \mathbb{Q} \cong \mathbb{L}\left(\widetilde{\mathrm{H}}_{*}(X ; \mathbb{Q})\right) .
$$

Example 5.3. Loop spaces. The loop space $\Omega X$ of any 1-connected space $X$ is a Koszul space with cohomology the free graded commutative algebra $\Lambda(V)$ on the graded vector space $V=\left(\pi_{*}(\Omega X) \otimes \mathbb{Q}\right)^{\vee}$. The Koszul dual Lie algebra is the abelian Lie algebra

$$
\pi_{*}\left(\Omega^{2} X\right) \otimes \mathbb{Q} \cong(s V)^{\vee} .
$$

Example 5.4. Products and wedges. If $X$ and $Y$ are Koszul spaces, then so are $X \times Y$ and $X \vee Y$. Indeed, it is easy to check that products and wedges of (co)formal spaces are (co)formal; see e.g., [25, Lemma 4.1]. On the level of cohomology, this is reflected by the fact that tensor and fiber products of Koszul algebras are Koszul:

$$
\begin{aligned}
& \mathrm{H}^{*}(X \times Y ; \mathbb{Q}) \cong \mathrm{H}^{*}(X ; \mathbb{Q}) \otimes \mathrm{H}^{*}(Y ; \mathbb{Q}), \\
& \mathrm{H}^{*}(X \vee Y ; \mathbb{Q}) \cong \mathrm{H}^{*}(X ; \mathbb{Q}) \times_{\mathbb{Q}} \mathrm{H}^{*}(Y ; \mathbb{Q})
\end{aligned}
$$

Example 5.5. Configuration spaces. For any $k$ and $n$ the configuration space $F\left(\mathbb{R}^{n}, k\right)$ of $k$ points in $\mathbb{R}^{n}$ is a Koszul space. This follows because configuration spaces are formal (see e.g., [17]) and their cohomology algebras are Koszul. The rational cohomology algebra is generated by elements $a_{p q}$ of cohomological degree $n-1$ for $1 \leq p<q \leq k$ subject to the 'Arnold relations'

$$
\begin{gathered}
a_{p q} a_{q r}+a_{q r} a_{r p}+a_{r p} a_{p q}=0, \quad p, q, r \text { distinct }, \\
a_{p q}^{2}=0, \quad(n \text { odd }) .
\end{gathered}
$$

Here we use the convention $a_{p q}=(-1)^{n} a_{q p}$ for $p>q$. This algebra has a PBWbasis [30] consisting of all monomials $a_{i_{1} j_{1}} \ldots a_{i_{r} j_{r}}$ where $i_{1}<\ldots<i_{r}$ and $i_{p}<j_{p}$ for all $p$, and therefore it is Koszul. Hence, we have an isomorphism of graded Lie algebras $\pi_{*}\left(\Omega F\left(\mathbb{R}^{n}, k\right)\right) \otimes \mathbb{Q}=\mathrm{H}^{*}\left(F\left(\mathbb{R}^{n}, k\right) ; \mathbb{Q}\right)^{!} \mathscr{L}_{i e}$. When calculating the orthogonal relations we recover [9, Theorem 2.3]: as a graded Lie algebra $\pi_{*}\left(\Omega F\left(\mathbb{R}^{n}, k\right)\right) \otimes \mathbb{Q}$ is generated by classes $\alpha_{p q}$ of homological degree $n-2$ for $1 \leq p<q \leq k$ subject to the 'infinitesimal braid relations' or 'Yang-Baxter Lie algebra relations'

$$
\begin{aligned}
{\left[\alpha_{p q}, \alpha_{r s}\right] } & =0, & & \{p, q\} \cap\{r, s\}=\emptyset, \\
{\left[\alpha_{p q}, \alpha_{p r}+\alpha_{q r}\right] } & =0, & & p, q, r \text { distinct. }
\end{aligned}
$$

Again, we use the convention that $\alpha_{q p}=(-1)^{n} \alpha_{q p}$ for $p>q$.

Example 5.6. Highly connected manifolds. Let $M$ be a $(d-1)$-connected closed $m$-dimensional manifold with $m \leq 3 d-2, d \geq 2$, and where $\operatorname{dim}_{\mathbb{Q}} \mathrm{H}^{*}(M ; \mathbb{Q}) \geq$ 4. By [25, Proposition 4.4] any such manifold is formal and coformal, whence a Koszul space by Theorem 1.2. By Poincaré duality and for degree reasons, the rational cohomology of $M$ admits a basis $1, x_{1}, \ldots, x_{n}, \omega$, where $\omega$ is a generator of $\mathrm{H}^{m}(M ; \mathbb{Q}) \cong \mathbb{Q}$ and where $x_{1}, \ldots, x_{n}$ are indecomposable with respect to the cup product. Therefore, the cohomology algebra is completely determined by the structure coefficients $q_{i j} \in \mathbb{Q}$ where

$$
x_{i} x_{j}=q_{i j} \omega .
$$


By Theorem 1.3, we can compute the rational homotopy Lie algebra of $M$ by finding the orthogonal relations. Clearly, a relation

$$
\sum_{i, j} c_{i j} x_{i} x_{j}=0
$$

holds if and only if $\sum_{i, j} q_{i j} c_{i j}=0$. So we get that $\pi_{*}(\Omega M) \otimes \mathbb{Q}$ is a free graded Lie algebra on classes $\alpha_{1}, \ldots, \alpha_{n}$ modulo a single quadratic Lie form

$$
\begin{gathered}
\pi_{*}(\Omega M) \otimes \mathbb{Q} \cong \mathbb{L}\left(\alpha_{1}, \ldots, \alpha_{n}\right) /(Q), \\
Q=\sum_{i, j}(-1)^{\left|x_{i}\right|\left|\alpha_{j}\right|} q_{i j}\left[\alpha_{i}, \alpha_{j}\right] .
\end{gathered}
$$

This recovers [24, Theorem 5] up to a sign.

Example 5.7. Davis-Januszkiewicz spaces associated to flag complexes. The DavisJanuszkiewicz space $D J(K)$ associated to a simplicial complex $K$, first introduced in [10], plays a central role in toric topology; see [7. Notbohm and Ray [26] have proved that $D J(K)$ is always formal. The cohomology algebra $\mathrm{H}^{*}(D J(K) ; \mathbb{Q})$ is isomorphic to the Stanley-Reisner algebra $\mathbb{Q}[K]$. If $K$ has vertices $v_{1}, \ldots, v_{n}$, this is a polynomial algebra on generators $x_{1}, \ldots, x_{n}$ of cohomological degree 2 modulo those monomial relations

$$
x_{i_{1}} \ldots x_{i_{r}}=0
$$

for which $v_{i_{1}}, \ldots, v_{i_{r}}$ do not span a face of $K$. According to [14, a graded commutative algebra with monomial relations is Koszul as soon as all relations are quadratic. Combined with the formality of $D J(K)$, and Theorem 1.2, this implies that $D J(K)$ is a Koszul space if and only if $K$ is a flag complex, i.e., if and only if all minimal non-faces of $K$ are of cardinality two. This recovers [27, Theorem 9.6]. An easy calculation of the orthogonal relations shows that if $K$ is a flag complex, then the homotopy Lie algebra $\pi_{*}(\Omega D J(K)) \otimes \mathbb{Q}$ is generated by classes $\alpha_{1}, \ldots, \alpha_{n}$ of degree 1 modulo the relations

$$
\left[\alpha_{i}, \alpha_{j}\right]=0, \quad i=j \text { or } v_{i}, v_{j} \text { span an edge of } K \text {. }
$$

\section{ACKNOWLEDGments}

The author is grateful to Alexandru Suciu for directing his attention to the paper 29. He also thanks Bruno Vallette and Joan Millès for discussions about Koszul duality for algebras over operads. Finally, the author would like to thank the referee for helpful comments.

\section{RefERENCES}

[1] David J. Anick, A counterexample to a conjecture of Serre, Ann. of Math. (2) 115 (1982), no. 1, 1-33, DOI 10.2307/1971338. MR644015(86i:55011a)

[2] David J. Anick, Comment: "A counterexample to a conjecture of Serre", Ann. of Math. (2) 116 (1982), no. 3, 661, DOI 10.2307/2007027. MR678485(86i:55011b)

[3] M. F. Atiyah and I. G. Macdonald, Introduction to commutative algebra, Addison-Wesley Publishing Co., Reading, Mass.-London-Don Mills, Ont., 1969. MR0242802 (39 \#4129)

[4] H. J. Baues and J.-M. Lemaire, Minimal models in homotopy theory, Math. Ann. 225 (1977), no. 3, 219-242. MR0431172 (55 \#4174)

[5] J. M. Boardman and R. M. Vogt, Homotopy invariant algebraic structures on topological spaces, Lecture Notes in Mathematics, Vol. 347, Springer-Verlag, Berlin, 1973. MR0420609 $(54$ \#8623a) 
[6] A. K. Bousfield and V. K. A. M. Gugenheim, On PL de Rham theory and rational homotopy type, Mem. Amer. Math. Soc. 8 (1976), no. 179, ix+94. MR0425956 (54 \#13906)

[7] Victor M. Buchstaber and Taras E. Panov, Torus actions and their applications in topology and combinatorics, University Lecture Series, vol. 24, American Mathematical Society, Providence, RI, 2002. MR1897064 (2003e:57039)

[8] F. R. Cohen, The homology of $\mathcal{C}_{n+1}$-spaces, $n \geq 0$, in "The homology of iterated loop spaces", Lecture Notes in Mathematics 533, Springer Verlag (1976), 207-351.

[9] F. R. Cohen and S. Gitler, On loop spaces of configuration spaces, Trans. Amer. Math. Soc. 354 (2002), no. 5, 1705-1748, DOI 10.1090/S0002-9947-02-02948-3. MR.1881013 (2002m:55020)

[10] Michael W. Davis and Tadeusz Januszkiewicz, Convex polytopes, Coxeter orbifolds and torus actions, Duke Math. J. 62 (1991), no. 2, 417-451, DOI 10.1215/S0012-7094-91-06217-4. MR 1104531 (92i:52012)

[11] Yves Félix, Stephen Halperin, and Jean-Claude Thomas, Rational homotopy theory, Graduate Texts in Mathematics, vol. 205, Springer-Verlag, New York, 2001. MR.1802847(2002d:55014)

[12] Yves Félix, John Oprea, and Daniel Tanré, Algebraic models in geometry, Oxford Graduate Texts in Mathematics, vol. 17, Oxford University Press, Oxford, 2008. MR2403898 (2009a:55006)

[13] Benoit Fresse, Koszul duality of operads and homology of partition posets, Homotopy theory: relations with algebraic geometry, group cohomology, and algebraic $K$-theory, Contemp. Math., vol. 346, Amer. Math. Soc., Providence, RI, 2004, pp. 115-215, DOI 10.1090/conm/346/06287. MR2066499 (2005g:18015)

[14] Ralph Fröberg, Determination of a class of Poincaré series, Math. Scand. 37 (1975), no. 1, 29-39. MR0404254(53 \#8057)

[15] E. Getzler, J. D. S. Jones, Operads, homotopy algebra and iterated integrals for double loop spaces, arXiv:hep-th/9403055v1

[16] Victor Ginzburg and Mikhail Kapranov, Koszul duality for operads, Duke Math. J. 76 (1994), no. 1, 203-272, DOI 10.1215/S0012-7094-94-07608-4. MR.1301191 (96a:18004)

[17] P. Lambrechts, I. Volic, Formality of the little $N$-disks operad, arXiv:0808.0457v2 [math.AT]

[18] Jean-Louis Loday and Bruno Vallette, Algebraic operads, Grundlehren der Mathematischen Wissenschaften [Fundamental Principles of Mathematical Sciences], vol. 346, Springer, Heidelberg, 2012. MR2954392

[19] Martin Majewski, Rational homotopical models and uniqueness, Mem. Amer. Math. Soc. 143 (2000), no. 682, xviii+149. MR.1751423(2001e:55015)

[20] J. P. May, The geometry of iterated loop spaces, Springer-Verlag, Berlin, 1972. Lectures Notes in Mathematics, Vol. 271. MR0420610 (54 \#8623b)

[21] Joan Millès, The Koszul complex is the cotangent complex, Int. Math. Res. Not. IMRN 3 (2012), 607-650. MR2885984

[22] John W. Milnor and John C. Moore, On the structure of Hopf algebras, Ann. of Math. (2) 81 (1965), 211-264. MR0174052 (30 \#4259)

[23] Joseph Neisendorfer, Lie algebras, coalgebras and rational homotopy theory for nilpotent spaces, Pacific J. Math. 74 (1978), no. 2, 429-460. MR494641 (80b:55010)

[24] Joseph Neisendorfer, The rational homotopy groups of complete intersections, Illinois J. Math. 23 (1979), no. 2, 175-182. MR.528555(80j:55018)

[25] Joseph Neisendorfer and Timothy Miller, Formal and coformal spaces, Illinois J. Math. 22 (1978), no. 4, 565-580. MR0500938(58 \#18429)

[26] Dietrich Notbohm and Nigel Ray, On Davis-Januszkiewicz homotopy types. I. Formality and rationalisation, Algebr. Geom. Topol. 5 (2005), 31-51 (electronic), DOI 10.2140/agt.2005.5.31. MR2135544(2006a:55016)

[27] Taras E. Panov and Nigel Ray, Categorical aspects of toric topology, Toric topology, Contemp. Math., vol. 460, Amer. Math. Soc., Providence, RI, 2008, pp. 293-322, DOI 10.1090/conm/460/09026. MR2428364(2009j:55026)

[28] Stefan Papadima and Alexander I. Suciu, Homotopy Lie algebras, lower central series and the Koszul property, Geom. Topol. 8 (2004), 1079-1125, DOI 10.2140/gt.2004.8.1079. MR2087079 (2005g:55022)

[29] Stefan Papadima and Sergey Yuzvinsky, On rational $K[\pi, 1]$ spaces and Koszul algebras, J. Pure Appl. Algebra 144 (1999), no. 2, 157-167, DOI 10.1016/S0022-4049(98)00058-9. MR:1731434(2000k:55017) 
[30] Stewart B. Priddy, Koszul resolutions, Trans. Amer. Math. Soc. 152 (1970), 39-60. MR.0265437(42 \#346)

[31] Daniel Quillen, Rational homotopy theory, Ann. of Math. (2) 90 (1969), 205-295. MR.0258031 (41 \#2678)

[32] Jean-Pierre Serre, Groupes d'homotopie et classes de groupes abéliens (French), Ann. of Math. (2) 58 (1953), 258-294. MR0059548 (15,548c)

[33] Jean-Pierre Serre, Algèbre locale. Multiplicités (French), Cours au Collège de France, 19571958, rédigé par Pierre Gabriel. Seconde édition, 1965. Lecture Notes in Mathematics, vol. 11, Springer-Verlag, Berlin, 1965. MR0201468 (34 \#1352)

[34] Dennis Sullivan, Infinitesimal computations in topology, Inst. Hautes Études Sci. Publ. Math. 47 (1977), 269-331 (1978). MR0646078 (58 \#31119)

[35] B. Vallette, Homotopy theory of homotopy algebras, preprint.

Department of Mathematical Sciences, University of Copenhagen, UniversitetsPARKEN 5, 2100 COPENhagen $\varnothing$, Denmark

E-mail address: alexb@math.ku.dk

Current address: Department of Mathematics, Stockholm University, SE-106 91 Stockholm, Sweden 